\title{
Gradhiva
}

GRADHIV

Revue d'anthropologie et d'histoire des arts

15 | 2012

Robots étrangement humains

\section{Le corps humain et ses doubles}

Sur les usages de la fiction dans les arts et la robotique

The human body and its doubles. About the uses of fiction in the arts and robotics

Joffrey Becker

\section{OpenEdition}

\section{Journals}

Édition électronique

URL : http://journals.openedition.org/gradhiva/2335

DOI : 10.4000/gradhiva.2335

ISSN : $1760-849 x$

\section{Éditeur}

Musée du quai Branly Jacques Chirac

\section{Édition imprimée}

Date de publication : 16 mai 2012

Pagination : 102-119

ISBN : 978-2-35744-047-0

ISSN : 0764-8928

\section{Référence électronique}

Joffrey Becker, « Le corps humain et ses doubles », Gradhiva [En ligne], 15 | 2012, mis en ligne le 16 mai 2015, consulté le 01 mai 2019. URL : http://journals.openedition.org/gradhiva/2335 ; DOI :

10.4000/gradhiva.2335 


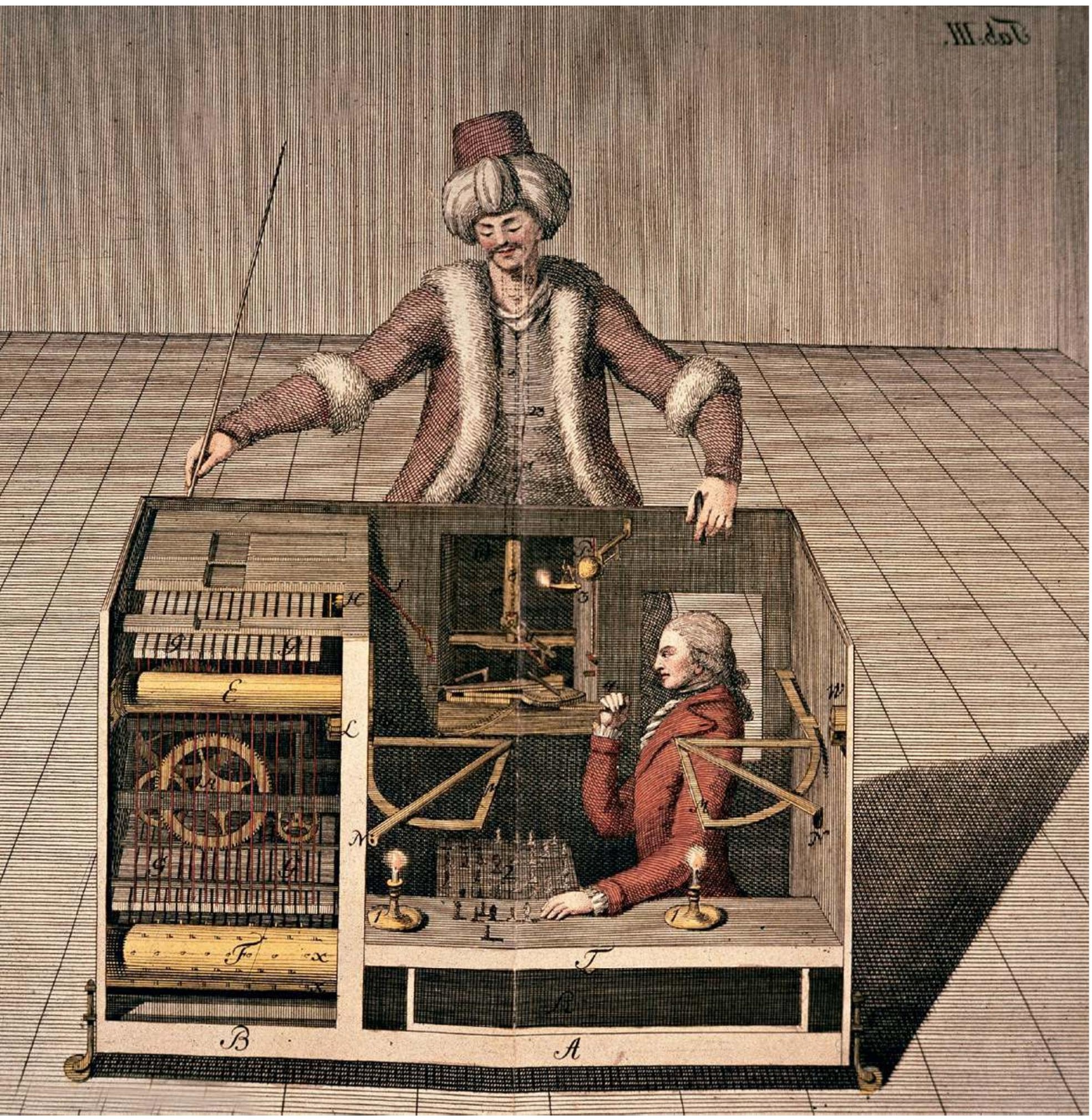

Fig. 1 Planche III, «Sur le joueur d'échecs de M. von Kempelen », Leipzig et Dresde : Joh. Gottl. Breitkopf, $1789 \odot$ Université Humboldt, Berlin/Bridgeman Giraudon. 


\section{Le corps humain et ses doubles Sur les usages de la fiction dans les arts et la robotique}

It is also widely assumed that to the engineer, scientist and mathematician, art is magic, and to the composer, painter and poet, technology is a mystery. Jasia Reichardt, Cybernetics, Art and Ideas, Greenwich, New York Graphic Society, 1971

Joffrey Becker ${ }^{*}$

\footnotetext{
- $\bullet$

"J'adresse mes remerciements à Carlo Severi, Denis Vidal et Emmanuel Grimaud, pour leur aide et les commentaires très profitables qu'ils formulent à l'égard de mon travail. Je remercie également Stelarc et David McGoran, ainsi que les membres du Bristol Robotics Laboratory, plus particulièrement les professeurs Alan Winfield, Chris Melhuish et Tony Pipe.

1. Le secret du joueur d'échecs est l'objet de nombreux traités et articles, dont un écrit par Edgar Allan Poe en 1836 pour le Southern Literary Messenger.
}

Lorsqu'en 1770 il adresse, depuis Presbourg, une lettre à l'éditeur du Mercure de France, Louis Dutens ne peut douter du génie de Johann Wolfgang von Kempelen, conseiller auprès de l'empereur et directeur des salines de Hongrie, qui présente alors son Turc dans une sorte de défi lancé à la noblesse européenne (Dutens 1772). Automate plus grand qu'un homme, monté sur un coffre où est posé un jeu d'échecs, le Turc est capable de jouer et de vaincre les meilleurs joueurs. Cette machine a pourtant de quoi susciter le doute. «Si M. de Kempel, écrit ainsi JeanAntoine Rigoley de Juvigny, dans une réponse parue dans le Mercure de France de décembre 1770, a pu donner à son automate des mouvements indéterminés, et dépendants d'une volonté quelconque et étrangère, il faudra regarder son auteur comme un homme surnaturel. » (Rigoley de Juvigny 1770 :187) En fait de prodiges surnaturels, le Turc consiste en une illusion savamment mise en œuvre par le mécanicien, "une machine qui n'est pas sans mérite du côté du mécanisme, mais dont les effets ne paraissent si merveilleux que par la hardiesse de l'idée, et par l'heureux choix des moyens que [l'Inventeur] emploie pour faire illusion " (Windisch $1783: 40-41$ ). Une illusion bien connue, dont Kempelen ne cache pas l'existence, mais dont le secret sera conservé jusqu'après sa mort. En réalité, si le Turc semble aussi doué pour le jeu d'échecs, c'est qu'il abrite un joueur humain dans le coffre qui forme sa base, habilement dissimulé aux yeux des spectateurs et de son adversaire. Hormis la très vive curiosité qu'il suscite, cet objet provoque des réactions très particulières, passant pour une machine diabolique, provoquant des sentiments de peur mêlés d'admiration ou entraînant les plus érudits dans de longues discussions sur l'origine de son fonctionnement ${ }^{1}$. 


\section{ROBOTS ÉTRANGEMENT HUMAINS}

-

2. Dès la fin du xvIII' siècle, Friedrich Nicolai note que l'automate n'est rien de plus qu'un moulin à café et que le procédé employé par Vaucanson relève d'une illusion, la nourriture ingérée par le canard restant à la base du tube descendant de son bec, et le canal d'entrée de la nourriture étant sans relation avec celui par lequel sort «l'excrément ». Le célèbre magicien, par ailleurs mécanicien et horloger, Jean-Eugène RobertHoudin a eu l'occasion de réparer le canard avant son installation à l'exposition des produits de l'industrie au Palais-Royal, à Paris, en 1844. Confirmant les propos de Nicolai, il ajoute : «Vaucanson n'était pas seulement mon maître en mécanique, je devais m'incliner aussi devant son génie pour l'escamotage. » (RobertHoudin 1859 : 248-250) Ces deux témoignages ont récemment été remis en cause par Jean-Claude Heudin, pour qui il est possible que le canard exposé à Paris en 1844 ait été un faux (Heudin 2008 : 57-61).

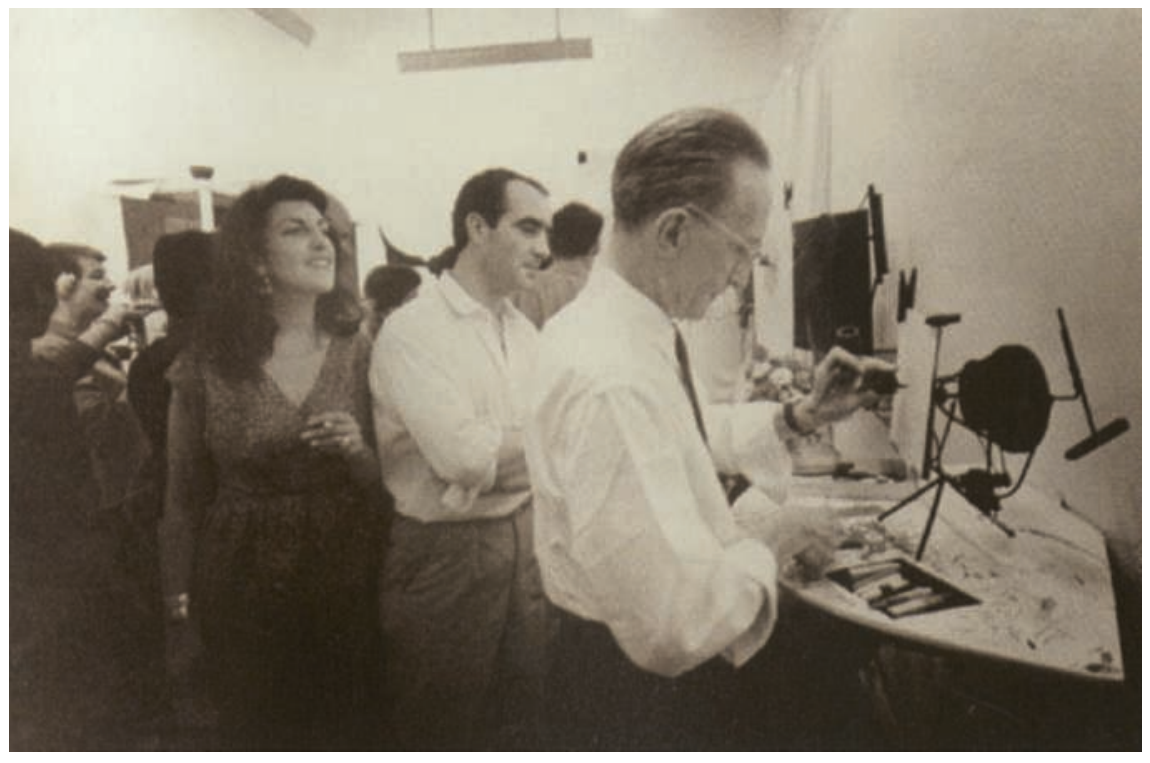

Fig. 2 Iris Clert, Jean Tinguely et Marcel Duchamp avec les méta-matics de Tinguely, galerie Iris Clert, Paris, 1959 @ ADAGP, Paris 2012, photo DR.

Pour Paul Metzner, cette manière de concevoir des automates emprunte pour beaucoup aux procédés de fabrication des tours de magie. Il note, par ailleurs, que la ferveur populaire pour les uns et les autres se développe à peu près à la même époque; et que mécaniciens et illusionnistes recourent à des techniques tout à fait analogues (Metzner 1998). Plus encore, l'illusion procéderait, alors, d'un mode spécifique de présentation au public. Les automates, par l'attraction et le divertissement qu'ils procurent, mais également par la manière dont ils simulent la vie, constitueraient des formes de dramatisation des questions philosophiques portant sur le caractère mécanique des fonctions vitales (Riskin 2003: 601). Le canard digérateur conçu par le célèbre mécanicien grenoblois Jacques de Vaucanson est, à ce titre, exemplaire. Le principe mécanique de digestion par dissolution illustré par cet automate (Vaucanson 1738:19) ne ressortit certes pas à une illusion du même ordre que celle élaborée par Kempelen. Le doute qui entoure cet objet révèle néanmoins les rapports très particuliers que nous entretiendrions avec les créatures artificielles. Il montre également toute l'ambiguïté du statut de la fiction dans les sciences².

L'incertitude et la croyance qui se mêlent, à divers degrés, dans les expériences que nous venons d'évoquer peuvent constituer un moyen pour comprendre une part des relations que nous entretenons aujourd'hui avec les «héritiers" des automates des XVIII ${ }^{\mathrm{e}}$ et XIX ${ }^{\mathrm{e}}$ siècles, les robots. On peut s'étonner de ce rapprochement entre la robotique et des techniques qui relèvent de processus fictionnels. Cette science, hormis les défis techniques qu'elle cherche à relever (et qui constituent le premier souci des ingénieurs), aspire en effet à produire une meilleure connaissance de l'humain par l'imitation de certaines de ses fonctions, données à voir à travers le comportement des objets. Il peut d'abord paraître difficile de la considérer par référence aux techniques employées par les illusionnistes. On sera peut-être moins étonné si l'on considère que l'emploi de la fiction fait converger des domaines aussi différents que les sciences et les arts dans une même dynamique, dont l'enjeu est à la fois une histoire et une transformation de nous-mêmes. Nous nous intéresserons d'abord à la manière dont l'usage de la 
robotique par les artistes permet d'inclure et d'orienter le public dans un espace relationnel. Nous verrons ensuite comment un tel espace peut être mobilisé en robotique, en nous appuyant sur une expérience portant sur une question récurrente du domaine : l'acceptation de la machine comme partenaire social. Enfin, nous mettrons en perspective ces deux manières d'envisager les relations entre humains et machines, en soulignant le rôle particulier que jouent l'ambiguité et la projection dans la convergence des arts et des sciences.

\section{La machine spectaculaire}

L'histoire des robots est d'abord celle des ingénieurs. L'intérêt pour la mécanique intègre néanmoins l'espace des arts dès le début du xxe siècle. Marcel Duchamp s'engage dans la sculpture cinétique avec sa Roue de bicyclette en 1913, puis avec Rotary glass plates en 1920 et Anemic cinema en 1926. C'est également dans les années 1920-1930 qu'Alexander Calder conçoit son Cirque et ses premiers mobiles. Mais il faut attendre les années 1950-1960, et l'essor de la cybernétique, pour que les premiers robots entrent dans les ateliers des artistes. Nicolas Schöffer réalise ainsi une sculpture spatiodynamique, CYSP-1, qui grâce à ses moteurs et ses capteurs de proximité peut accompagner et réagir aux mouvements des dan-

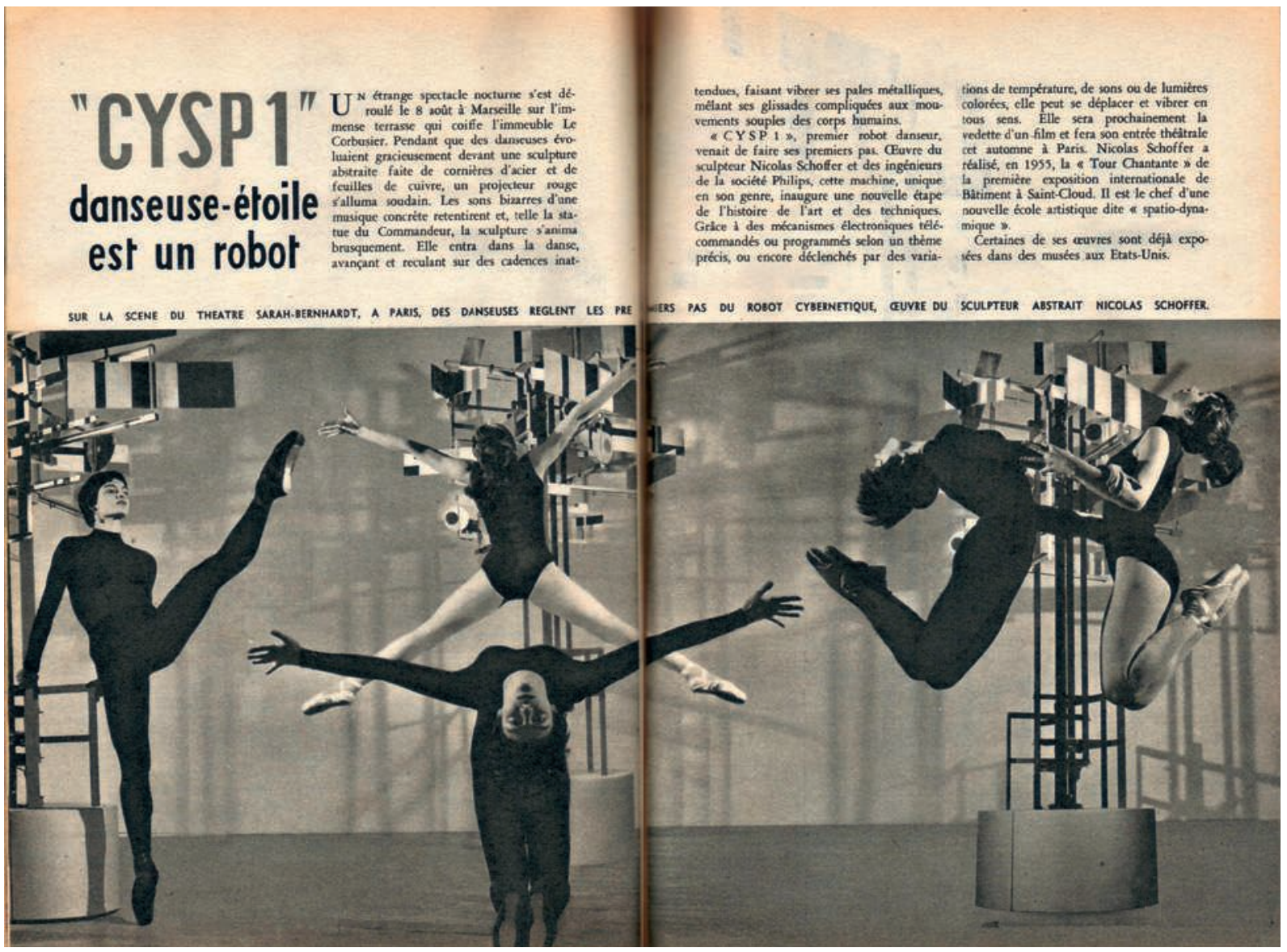

Fig. 3 Nicolas Schöffer, CYSP-1, In Sciences \& Vie, sept. 1956. Avec l'aimable autorisation de Reuben Hoggett et d'Eléonore de Lavandeyra Schöffer. 


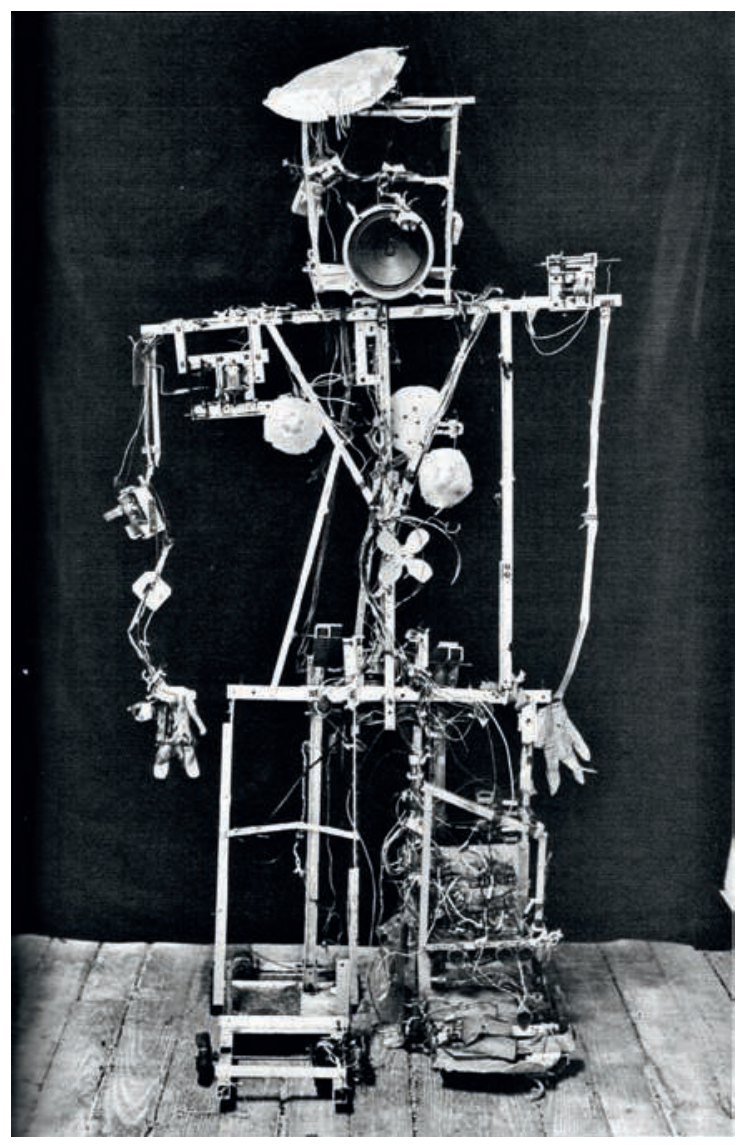

seuses de Maurice Béjart sur le toit de la Cité radieuse, à Marseille, en 1956. C'est également à cette époque que Jean Tinguely construit ses Machines à dessiner et ses Méta-Matics.

En 1964, Nam June Paik conçoit un robot téléopéré à taille humaine (Dixon 2007: 284), une machine qu'il nomme Robot $K-456$ en référence au dix-huitième concerto pour piano de Mozart, portant le numéro 456 dans la classification de Köchel. Les premières actions de ce robot ont lieu dans le cadre de l'Annual New York Avant-Garde Festival, d'abord au Judson Hall, puis dans la rue. Cette machine télécommandée, dont l'humanité était formellement réduite à quelques traits saillants - une tête en boîte vide rappelant les hommes cubiques de Giovanni Battista Braccelli (1624), une armature de fer dessinant grossièrement les bras, le torse et les jambes -, imposait un double point de vue, politique et ironique. Par le haut-parleur lui servant de bouche, elle diffusait le discours d'investiture de John F. Kennedy. Rappelant le canard de Vaucanson, elle excrétait des grains. Paik, souligne Eduardo Kac, cherchait à mettre en valeur les notions de contrôle à distance et de mobilité à travers une interaction directe avec le public (Kac 1997). Mais son objet avait de quoi faire sourire. Pour Paik, il fallait considérer le robot comme une caricature et non comme la source d'une angoisse, la machine remplaçant le corps humain étant d'une fragilité telle qu'elle réclamait la vigilance constante de trois ou quatre personnes. Ainsi, dans un texte où il rend hommage à l'ingénieur Shuya Abe, qui l'aidera notamment à concevoir son synthétiseur vidéo, il souligne :

Fig. 4 Nam June Paik et Shuya Abe, K-456, 1964, DR Photo (C) Estate of Peter Moore/ VAGA, NYC.
Le robot que j'ai fabriqué avec M. Kyuta Saito, étudiant à l'Electric Communication College, est tombé en morceaux après un premier essai. Suivant les conseils de $M$. Abe, $M$. Saito a remis en place tout le mécanisme et, finalement, le robot a pu se déplacer en titubant. Peu après mon arrivée à New York, ce Robot $K$-456 a pris feu sur Canal Street et j'ai donc dû remplacer le circuit de relais moi-même. Ensuite, il a fait une brève apparition lors de l'ouverture du troisième Festival d'avant-garde durant la performance de Charlotte Moorman. Voilà comment j'ai utilisé le million de yens que mon frère m'avait donné à Tokyo. (Decker et Leeber 1993 : 35)

Paik mit finalement en scène la fin de sa machine en 1982 lors d'une rétrospective : Catastrophe of technology in the Twenty-First Century (Hanhardt 2006). Il lui fit quitter le trottoir sur lequel elle évoluait, et le robot fut renversé par une voiture. On le ramena ensuite à son lieu d'exposition. Auparavant, Paik avait également présenté Robot $K-456$ lors de l'exposition Cybernetic Serendipity, organisée à Londres en 1968. Conçue par Jasia Reichardt, celle-ci réunissait plus de trois cents ingénieurs et artistes, parmi lesquels Bruce Lacey et Tinguely, autour de l'idée que l'humain pouvait étendre sa créativité et son inventivité grâce à la technologie et aux ordinateurs. Elle accueillait également la sculpture cinétique d'Edward Ihnatowicz, SAM (Sound Activated Mobile). SAM était sensible aux événements sonores de son environnement. L'objet consistait en une série de vertèbres d'aluminium parcourues de pistons hydrauliques et surmontées par une sorte de fleur en fibre de verre dont les pistils étaient formés par quatre microphones. Un circuit électronique traitait les signaux reçus et permettait d'orienter la structure en fonction de l'endroit d'où provenait le son et de sa puissance (Zivanovic 2005). Le robot laissait ainsi penser qu'il suivait les mouvements des 
spectateurs passant à proximité ou réagissait en fonction des sons produits face à lui. Ce comportement très simple fascina l'assistance, et certains spectateurs jouèrent avec cette machine pendant des heures, lui parlant, changeant de place et lui parlant encore, riant de temps en temps (Reichardt 1972).

Les robots comme $K-456$ ou SAM ont, il est vrai, quelque chose d'amusant. Cet amusement semble lié à leur façon d'impliquer le public dans la situation particulière créée par leur présence. Néanmoins, les formes ludiques d'interaction ne sont pas la seule source d'expérimentation pour l'art. Des travaux plus récents ont mis l'accent sur le développement d'une relation beaucoup moins divertissante. Ainsi, dès le début des années 1970, l'artiste australien Stelarc a cherché à faire de la tentative d'hybridation de son corps avec l'objet technique un événement spectaculaire. Cette fusion de l'artiste et de son œuvre constitue en réalité un trait caractéristique de l'art de la performance. Mais il est ici réexploré, jouant avec l'inquiétante étrangeté, à la manière des travaux de Robert Whitman³, mais aussi avec l'empathie envers un corps dont la souffrance est rendue publique, comme, entre autres, dans ceux de Gina Pane ${ }^{4}$.

S'il est une constante dans l'œuvre de Stelarc, elle se trouve peut-être au sein de la relation très intime que celui-ci entretient avec les technologies qu'il emploie. Certaines machines incorporent des éléments de son image humaine (Prosthetic Head, 2002-2003). D'autres s'intègrent à sa chair (Stomach Sculpture, 1993). Parfois son corps commande leurs mouvements (Muscle Machine, 2003), parfois ce sont elles qui « décident » des gestes que l'artiste doit accomplir5 (Movatar, 2000). Lucy Suchman note que Stelarc ne dote pas ses objets d'une agentivité par le seul transfert de sa propre capacité d'action. Ses expériences jouent en réalité sur un subtil agencement entre la faculté qu'auraient les technologies à vider le corps de toute volonté et l'ambition de garder le contrôle sur ces dispositifs techniques. Son œuvre vise ainsi à donner consistance à des formes d'assemblage entre l'artiste, les technologies qu'il mobilise et le public, dans le temps et l'espace de la performance (Suchman 2007 : 255).

Le travail de Stelarc peut être compris comme une fiction idéologique. Le dispositif scénographique se construit sur la confusion entre le corps et l'objet technique, grâce à l'utilisation de technologies qui étendent l'activité corporelle autant qu'elles en prennent possession. Il met en forme une dénaturation du corps (Dixon 2007: 321) dont le spectateur est à la fois le témoin et l'acteur. Mais cette métamorphose n'est que temporaire. Elle repose sur le truchement de l'image générale présentée au public, mais aussi sur la manière dont ce dernier peut être intégré au processus de création de l'œuvre. Ces modalités de la confusion des corps humains et des objets sont en réalité assez caractéristiques de l'art de la performance (Kirby 1965). Elles sont clairement illustrées dans le travail de l'artiste australien. Dans Exoskeleton, par exemple (fig. 5), il se fait l'opérateur d'une machine marchante conçue par le F18 Institute for Art Information and Technology. Il porte des vêtements noirs et une prothèse robotique dissimule son bras droit. Derrière lui, une représentation aplanie de l'ensemble est montrée; une ombre qu'un puissant projecteur permet d'envoyer sur le mur, à laquelle s'ajoute parfois un écran diffusant l'image d'une caméra fixée au plafond. La chimère, mixte d'humain et d'insecte qui rappelle les figures mythologiques des théranthropes (Dixon 2007 : 320), évolue alors dans l'espace. Les déplacements de l'exosquelette sont limités par le câblage qui le lie à la régie, mais l'artiste, lors de cette performance, n'hésite pas à aller vers les spectateurs, à les frôler, dans le vacarme amplifié que produit la machine lorsqu'elle est
-

3. Dans The American Moon, happening présenté à la galerie Reuben de New York en 1960, les spectateurs sont invités à considérer les mouvements de balles de tissu, sortes de marionnettes parfois habitées par le corps d'un acteur (Kirby 1965 : 142-143).

4. Dans Le Lait chaud (1972), Gina Pane entaille son dos et ses joues avec une lame de rasoir, déclenchant des réactions d'empathie très vives parmi les spectateurs (Jones et Warr 2005 : 121).

5. Pour un aperçu de l'œuvre de Stelarc, on se référera à l'ouvrage dirigé par Marquard Smith (2005). 


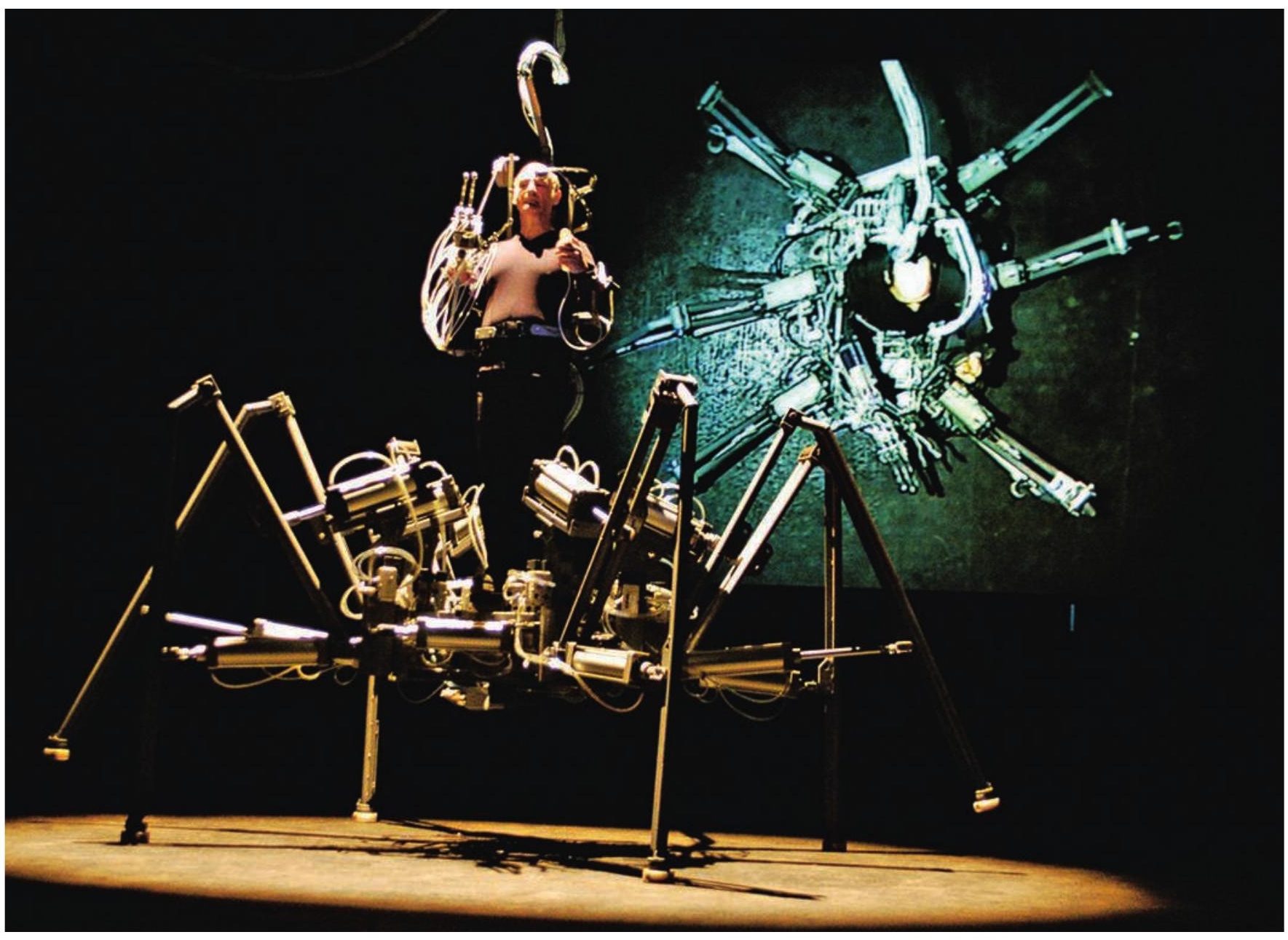

Fig. 5 Stelarc, EXOSKELETON. Cankarjev Dom, Ljubljana 2003. Photo Igor Skafar.

-

6. La recherche conduit depuis le milieu des années 1960 des travaux concernant le développement d'exosquelettes robotisés (comme Hardiman, développé par General Electric à partir de 1965) ou le contrôle à distance des comportements (Delgado 1969). en mouvement. Parfois, le public se sent directement menacé par l'imposante structure de six cents kilos et préfère se déplacer afin d'éviter tout contact avec elle. Dans d'autres cas, comme Fractal Flesh et Ping Body (fig. 6), le public est le complice, conscient ou non, de l'animation du corps même de l'artiste. C'est à lui qu'il revient de sélectionner le membre à animer, depuis une console ou en effectuant une action sans rapport direct avec la situation (comme une requête sur Internet), qui est automatiquement traitée par une machine tierce, puis convertie en signaux qui sont finalement envoyés sous forme d'impulsions électriques vers les muscles du performer.

Cette construction mutuelle de l'œuvre se traduit par une forte continuité entre chaque élément du dispositif. Elle évoque d'abord la tradition mécaniste chère aux fabricants d'automates, mais comporte également une dimension sociale naissant du croisement des actes déterminés par l'artiste et du rapport d'indétermination entre le public et les machines. D'une part, les dispositifs inventés par Stelarc rendent visibles des formes d'expérimentation ayant cours dans le domaine des sciences. Ils consistent en une mise en scène de la recherche ${ }^{6}$. D'autre part, en déplaçant l'expérience sur son corps, ils s'inscrivent dans une esthétique radicale. La performance constitue alors un moyen de 
capter l'attention des spectateurs et de les aider à penser une transformation à laquelle ils prennent directement part? 7 . Finalement, dans l'esthétique construite par l'artiste, la machine permet une profonde remise en question de l'humanité des éléments pris dans le dispositif, mais cette dernière ressortit toujours à une initiative humaine, que celle-ci soit volontaire ou qu'elle dépende du traitement stochastique des signaux émis par l'activité des humains, sans même qu'ils s'en rendent compte. Cette opposition entre les actes volontaires et involontaires, conscients et inconscients, n'est d'ailleurs pas sans rappeler quelques principes relatifs à la distinction entre les notions d'autonomie et d'automatisme dans la danse (Suquet 2006; Franko 1993: 19). Elle vient souligner la dimension chorégraphique de l'œuvre de Stelarc et, à travers elle, la disposition du public à s'engager dans l'œuvre. Car, au fond, la robotique de Stelarc induit des réponses émotionnelles assez diverses parmi les spectateurs, hésitant entre l'admiration et la crainte. Toutefois, cette variation des sentiments induits par l'artiste est traversée par une idée; celle de la possibilité d'un avenir où le corps et l'objet technique auraient fusionné.

Il me semble inutile de chercher à savoir si Stelarc est une sorte de savant fou ou un visionnaire. Son œuvre me semble bien plus intéressante si on l'aborde par le biais de ses qualités techniques, de ses nombreuses références aux méthodologies et à l'histoire de la performance, ou de sa propension à nous projeter dans l'idée que nous nous faisons du futur. Or nous avons vu jusqu'ici que la robotique, dans l'art, intervient comme une technique visant à construire non seulement
- -

7. Cette forme de réflexivité est un élément clé de la définition de la notion de performance pour Victor Turner (Turner 1987 : 81).

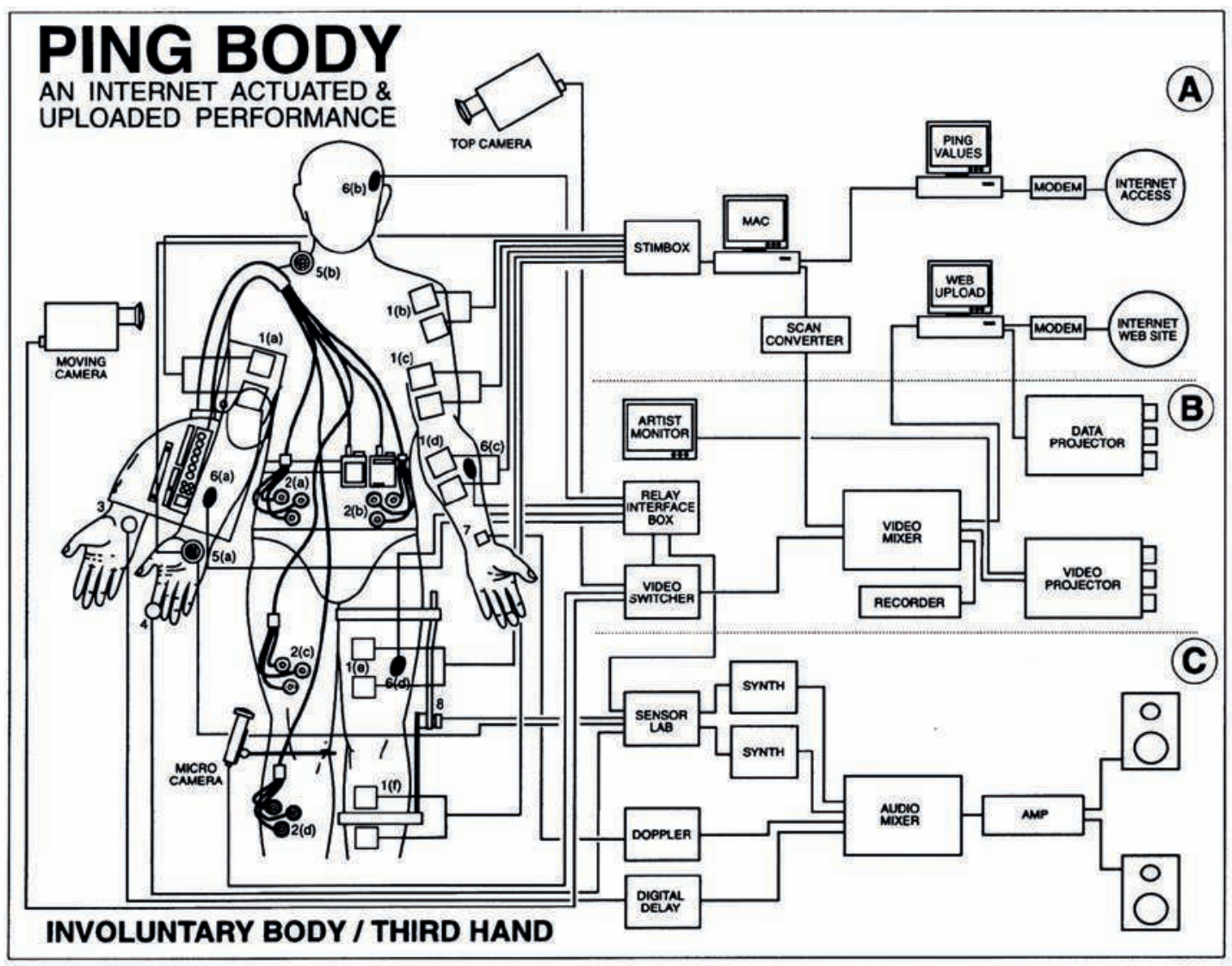

Fig. 6 Stelarc, INVOLUNTARY BODY/THIRD HAND. Yokohama, Melbourne 199o. Diagrammme Stelarc. 


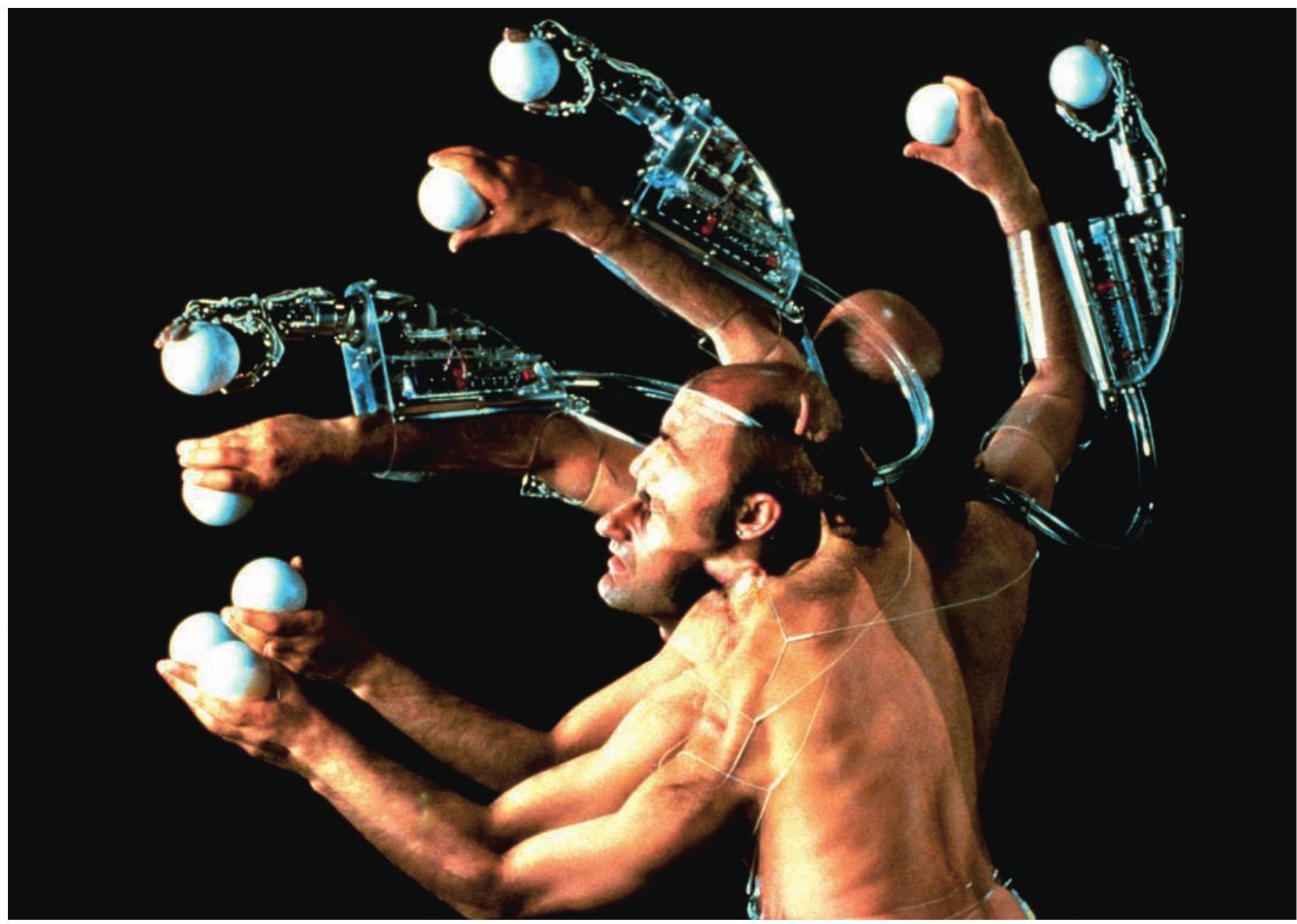

Fig. 7 Stelarc, MULTIPLE HANDS, Roppongi Studio, Tokyo, 1982. Photo T. Ike ๔ Stelarc.

\section{-}

8. Celle-ci consiste à faire passer pour opératoire une métamorphose n'existant pourtant que lors de la performance.

9. Un des cas les plus emblématiques de ces emprunts à l'art est celui de la robotique des émotions. L'expression des émotions par une machine virtuelle ou humanoïde tient d'une double représentation : d'une part, elle dépend de la manière dont les fonctions émotionnelles humaines ont été comprises; d'autre part, elle repose sur une iconographie de la représentation des émotions. Sur ce point, une lecture croisée du Traité des passions de l'âme de René Descartes (1649) et de la Conférence sur l'expression des passions par Charles Le Brun (1668) peut être assez éclairante. un dispositif scénographique, mais également, et surtout, un moyen de susciter la fascination des spectateurs en capturant leur attention (Gell 1998: 69). En nous informant ainsi sur la façon dont le public est invité à s'engager dans l'image qui lui est offerte, la fiction ${ }^{8}$ forme un espace pour l'expérimentation. Elle devient un moyen d'étudier les interactions entre humains et machines.

\section{La robotique des comportements et des émotions}

"Construire des robots, écrit Frédéric Kaplan, c'est à la fois pratiquer la magie, inventer des solutions technologiques nouvelles et comprendre l'homme. " (2005: 167) Dans son souci d'amener des preuves de la mécanicité de l'humain, la robotique, à travers la somme importante d'objets qu'elle produit, a non seulement recours à la performance, mais aussi, et plus curieusement, à l'illusion. Elle use ainsi, volontairement ou non, de pratiques et de méthodes relevant de l'art ${ }^{9}$. On peut déjà noter que les roboticiens conçoivent parfois des protocoles d'expérimentation où l'utilisateur est, à son insu, mis en présence d'une machine commandée, non par son programme, mais par un opérateur humain. Cette technique, dite 
du Magicien d'Oz, ressemble beaucoup à l'illusion élaborée par Kempelen. Elle offre notamment aux chercheurs qui s'intéressent aux interactions humainsmachines une occasion d'évaluer comment une technologie en cours de développement, ou encore inexistante, sera reçue par de futurs utilisateurs. Mais ce mode particulier de création d'artifices ne nous intéressera pas ici. J'aimerais pénétrer plus avant dans la compréhension des systèmes relationnels tels que nous venons de les aborder dans le cadre de l'art et de la performance. Pour cela, je m'appuierai sur une expérience singulière menée au sein d'un important laboratoire de robotique britannique. L'objectif principal de cette recherche n'est pas de créer la réplique exacte d'un humain, mais plutôt de voir comment un humain considère une étrange créature avec laquelle il ne partage que quelques traits; une créature envers laquelle on éprouverait une sorte d'empathie résultant du contrôle exercé par l'opérateur et émergeant d'une relation à trois où chaque comportement individuel est pris dans une sorte d'improvisation généralisée.

David McGoran, qui est à l'origine de ce projet, s'y est dévoué durant quelques mois avec une équipe pluridisciplinaire comprenant des roboticiens et des artistes, lors de ses études en robotique. Il a d'abord été formé aux arts de la performance, à la danse, au théâtre, au mime et au masque. Il s'est par ailleurs beaucoup intéressé au théâtre de marionnettes, au cinéma, aux animatroniques, et s'est finalement tourné vers la robotique, qu'il enseigne aujourd'hui à l'université, au sein d'un département d'ingénierie et de design. Ce parcours est assez singulier. Beaucoup d'ingénieurs ont un vif intérêt pour les arts, mais peu d'entre eux les investissent directement, par la pratique. Parallèlement, si de nombreux artistes s'intéressent aux robots, ils ne semblent pas toujours très concernés ou même au courant des problèmes abordés par la robotique. McGoran, bien qu'il ne revendique pas le titre d'artiste, est de ceux (ils sont de plus en plus nombreux) qui voient un intérêt à réunir les deux domaines. C'est là la singularité de son approche. Mais lui-même souligne que cet intérêt croisé n'a absolument rien d'original et qu'on se méprend à considérer comme différentes deux façons pourtant très semblables d'envisager le travail de création.

Le projet Heart Robot se concentre principalement sur l'interaction humainmachine et repose sur deux questions très simples. Pouvons-nous aimer un robot? Un robot peut-il nous aimer en retour? McGoran, qui cultive ce goût pour la simplicité cher à Rodney Brooks ${ }^{10}$, a cherché à concevoir une interface rudimentaire, capable de peu d'actions, mais engendrant de grands résultats. Le robot (fig. 9) est équipé de quelques détecteurs l'aidant à déterminer sa position ou lui signalant la présence d'un objet dans sa main. Il cligne des yeux et simule la respiration. Une petite lumière rouge clignote sur son torse plus ou moins rapidement suivant l'état interne qu'il s'agit de figurer, selon que le robot est contracté (pulsation et respiration rapides) ou détendu (pulsation et respiration lentes). Cette alternance du rythme, la manière dont elle est déclenchée lors de l'interaction et dont elle trouve sa place au sein d'un groupe d'actions réflexes simulant des changements de l'état interne de la machine participent d'un scénario de base qui vise à créer l'illusion d'une réaction et, par conséquent, à faire émerger des sentiments d'empathie chez l'utilisateur humain.

Mais ce n'est pas tout. McGoran souligne que ce projet a été conduit sur une très courte période et qu'en conséquence le robot Heart n'a pu être complètement achevé. Le roboticien envisage d'ailleurs de poursuivre le travail afin de donner au robot une forme moins rudimentaire. Toutefois, on peut supposer que le caractère inachevé de cette machine renforce l'attachement observé pendant

\section{- $\bullet$}

10. Considéré comme l'un des pères de la robotique, Rodney Brooks a amorcé un tournant en intelligence artificielle, en reconsidérant notamment l'approche computationnelle symbolique. Pour lui, les systèmes vivants doivent être compris dans le monde qu'ils occupent et qui les fait réagir. Aussi, la conception de machines autonomes doit s'appuyer sur la perception artificielle de l'espace et des événements qui y ont cours. Cette nouvelle intelligence artificielle, souligne Brooks, repose sur l'émergence de comportements dépendant de l'interaction de petites unités comportementales. Elle compte sur des architectures sensorimotrices, dites de subsomption, dont la combinaison des éléments permet l'apparition de comportements complexes et adaptatifs (Brooks 1990). 
-

11. La mécanisation du corps occupe en effet une place ambiguë dans l'histoire de la modernité. Elle formule une vision d'un humain conçu et géré à la manière d'une machine, indissociable en ce sens des formes d'organisation politique, économique, sociale et technique de l'activité de travail (Schaffer 1999). L'idée qu'une telle technicisation du corps puisse conduire à l'élaboration de mécanismes surpassant l'humanité a été investie par les artistes dès le début du $x x^{e}$ siècle, notamment dans la pièce de théâtre Rossum's Universal Robot écrite par Karel Čapek en 1920, où le terme «robot » fait pour la première fois son apparition.

12. C'est là un des principaux enjeux du talent de l'opérateur, comme le souligne Donald Keene (1990 [1965]).

13. Le metteur en scène et activiste brésilien a profondément remis en cause la notion de spectateur. Sa vision du théâtre repose sur la nécessité que le spectateur se libère de ses chaînes, agisse et devienne lui-même un acteur (Boal 1996).

14. J'ai eu l'occasion de prendre la mesure de l'empathie qui en résultait et des effets de l'induction psychomotrice lors d'une collaboration avec des roboticiens de la société Aldebaran Robotics portant sur l'expression d'émotions par le robot Nao (Monceaux et al. 2009). l'expérience. Aux yeux de la plupart des spectateurs qui l'ont approché, Heart a l'air d'une créature fragile, malade, étrange, qui contraste avec l'image menaçante caractérisant les humanoïdes ${ }^{11}$. Par ailleurs, il ne peut se déplacer seul. Il est forcément associé à son opérateur humain. En tant que marionnettiste, McGoran possède une grande expérience de la narration d'histoires. Il a par ailleurs été formé à la tradition des marionnettes japonaises bunraku, ce qui lui donne une certaine aisance dans la manipulation dite à vue. Pour lui, cette technique d'animation a quelques points communs avec la robotique. Il y a dans cette tradition, souligne-t-il, un certain art de la gestion du hasard et de l'erreur, la marionnette étant autant dirigée par son manipulateur qu'elle le dirige elle-même ${ }^{12}$. L'effet dépend également de l'effacement de l'opérateur, paradoxalement visible du public - art d'attirer le regard du public sur le robot et son histoire, en somme.

La performance implique par ailleurs, et dans la continuité des expériences théâtrales d'Augusto Boal ${ }^{13}$, une participation active du public. Le résultat de cette rencontre avec la machine, et surtout les émotions qui en émergent, laisse supposer que l'empathie qu'éprouvent les humains naît de la remémoration d'une expérience vécue. Ainsi, lorsqu'il est manipulé, Heart est capable de montrer des signes de contraction. Sa "respiration", qui se manifeste par des oscillations de son ventre et une pulsation lumineuse sur sa poitrine, s'accélère. Il peut être placé dans les bras d'un spectateur. Le manipulateur prend soin d'orienter la tête de la machine de sorte qu'elle croise le regard de celui qui la tient. Elle cligne alors des yeux. Si le spectateur le tient immobile, le robot va ensuite montrer des signes de décontraction; la pulsation lumineuse visible sur son torse ralentit progressivement, ainsi que les oscillations de son ventre. Si l'on place ses doigts au creux de sa main, le robot les détecte et les enserre par réflexe, un peu comme le ferait un nouveau-né. Ces signes élémentaires, McGoran les tient de son expérience de marionnettiste. Ils s'organisent autour de trois principes : l'alternance entre la tension et la relaxation, la réaction à des stimuli de l'environnement (dont le sursaut constitue un des signes les plus évidents ${ }^{14}$ ) et la respiration. Dans cette situation, et ainsi doté d'éléments de base formant autant de signes d'une présence autonome mais d'une très grande fragilité, le robot fait directement référence au monde de la petite enfance. Le lien que le marionnettiste entretient avec lui est également celui d'un père à son enfant, d'un point de vue métaphorique (en tant qu'élément du dispositif fictionnel) et de manière littérale (il en est l'auteur). "L'objet est un peu comme un miroir ", dit le roboticien, quelque chose sur lequel il projette des éléments de lui-même.

Heart intervient en fait comme le support d'éléments narratifs assez divers. Le robot est formé d'éléments composites qui dépendent d'abord de son créateur; il est la matérialisation d'une histoire de la robotique que David conte et, à travers elle, celle de sa propre histoire dans la robotique. Mais ces éléments renvoient également aux liens que les spectateurs, enfants comme adultes, peuvent entretenir avec une représentation imaginée, remémorée, dont le robot forme un appui. Ce dernier fait émerger des souvenirs, des envies, et peut-être d'autres choses encore. «Le robot est l'objet d'une relation d'enfant à parent. Quand je le présente aux gens, il est dans mes bras et je le place dans les bras des spectateurs. La réaction d'attachement est presque systématique. Une fois, j'ai vu un homme, assez fort, fondre en larmes lorsqu'il a eu le robot dans les bras. Ces choses très touchantes font partie intégrante du projet Heart Robot. » Il est nécessaire d'apporter quelques précisions concernant l'usage, en pareil cas, de ce qu'on désigne sous le terme d'«émergence ». La robotique, notamment le domaine de 


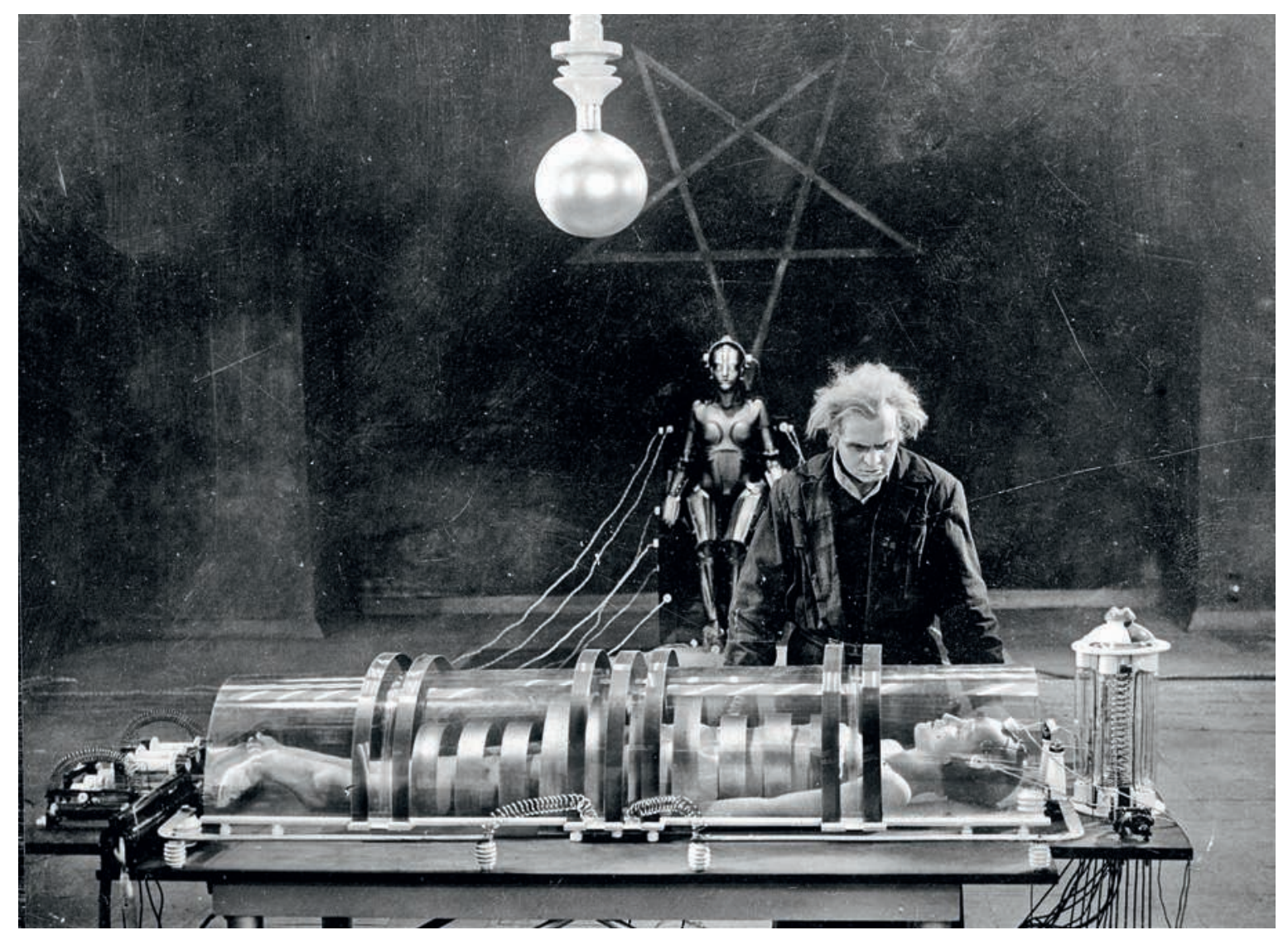

Fig. 8 Fritz Lang, Metropolis, avec Rudolf Klein-Rogge et Brigitte Helm, 1926 @ Roger-Viollet.

la vie artificielle, s'est récemment emparée de ce thème afin de comprendre comment l'autonomie d'un système peut être générée par l'ensemble des processus sous-jacents qui le composent (Bedau 1997). Suivant cette approche, la fiction construite par McGoran et son équipe invite à réfléchir à la manière dont les interactions entre l'opérateur, sa machine et le spectateur permettent de faire converger les éléments constitutifs de la performance vers une forme d'attachement. L'empathie qui semble caractériser la relation du public au couple «pèreenfant " que forment le marionnettiste et son robot apparait ainsi comme une émotion résultant directement du dispositif fictionnel. McGoran n'a aucun mal à reconnaitre le caractère construit de l'ensemble du dispositif. Celui-ci est d'ailleurs bien visible lors de l'interaction. Cette fiction constitue pourtant une expérience à part entière, portant sur les éléments minimaux d'une interaction entre un humain et un robot, soit, en d'autres termes, sur une façon dont les humains conservent le souvenir de leur attachement aux autres à travers l'expérience qu'ils ont des choses ${ }^{15}$ (Turkle 2007).

Une heuristique de la fiction

À l'heure où la simulation informatique tend à constituer un mode privilégié de l'expérience scientifique, il me semble nécessaire de lever certaines ambiguïtés quant au statut de la fiction tel que je l'ai abordé jusqu'à présent. J'ai

-

15. Sherry Turkle considère que les objets nous engagent dans une dynamique réflexive et émotionnelle, notamment par la manière dont ils sont entrés dans nos vies, la façon dont ils nous renvoient à des moments d'intimité, des époques ou des souvenirs. Certains, cependant, fondent leur puissance d'évocation sur leur étrangeté, c'est-à-dire sur le rapprochement qu'ils nous invitent à faire mentalement avec des formes que nous connaissons. 
Fig. 9 David McGoran, Heart Robot. Avec l'aimable autorisation de David McGoran, photo Ben Dowden.

\section{$\bullet \bullet$}

16. Les roboticiens semblent garder une certaine distance avec cet objet. Il est tour à tour considéré comme une «grande imposture 》 (Kaplan 2005: 165), « une mystification d'illusionniste 》 (Heudin 2008 : 70) ou encore désigné comme «le fameux faux automate » (Guillot et Meyer 2008:61]. tenté de montrer de quelles manières les objets robotiques participent d'un espace relationnel. Sur le mode du jeu, à travers l'anticipation d'une fusion du corps et de la machine ou la remémoration d'une expérience personnelle renvoyant à l'enfance, nous avons pu voir que l'intervention de robots auprès des spectateurs implique des projections qui dépendent principalement de ce qui est figuré par leurs concepteurs. Ces expériences s'inscrivent dans une perspective qui fait ainsi converger le monde des arts et celui des sciences. L'ancrage de la robotique dans la pratique artistique n'est toutefois pas si évident à penser de la part des roboticiens. Comme le souligne Kaplan, la différence fondamentale entre un savoir qui relèverait de la magie et un savoir scientifique réside en la prétention du second à une totale transparence. L'opacité qui néanmoins caractérise la perception du fonctionnement des machines induit nombre d'incertitudes concernant les actions dont elles sont réellement capables. Il est ainsi très simple de créer l'illusion de ces capacités en omettant d'en préciser les limites (Kaplan 2005 : 171-173), très simple de taire ce qui peut alors constituer la technologie d'une croyance. En ce sens, le type d'expérience qu'illustre le Turc automate de Kempelen apparaîtrait en complète contradiction avec la recherche de vérité caractérisant la tradition scientifique ${ }^{16}$.

La robotique emprunte des voies qui ne ressortissent pourtant pas toujours aux sciences. Véritable nœud de convergence entre les disciplines, elle reste d'abord difficilement accessible, y compris parfois pour ses initiés. L'opacité qu'elle produit ne doit toutefois pas être comprise comme une simple tentative d'abuser le public, même si elle apparaît de prime abord comme le résultat d'un savant mélange de technologie de l'enchantement et d'enchantement de la technologie (Gell 1992). Les robots offrent en réalité de nouvelles méthodes d'expérimentation, de nouvelles manières d'envisager les problèmes qui touchent à notre humanité. Malgré les limites de leur ressemblance avec les corps qu'ils imitent, ils forment autant de moyens d'un savoir sur nous-mêmes; une anthropologie qui, paradoxalement, construirait la compréhension de ce qui fonde notre humanité par le redoublement incertain de son image.

Il y a, dans les expérimentations que nous avons abordées, un moment clé où les robots sont pris pour autre chose que ce qu'ils sont en réalité, qu'il s'agisse du jeu que la machine propose au spectateur si l'on songe à $S A M$, des tensions qu'elle provoque si l'on pense au travail de Stelarc ou de la mémoire d'un état émotionnel qu'elle mobilise, comme nous l'avons vu avec Heart. À ce momentci, où l'illusion semble pourtant à son comble, a lieu un événement intéressant pour le chercheur : l'occasion lui est offerte d'accéder à l'expérience subjective du spectateur, d'en saisir la portée. L'alternance entre ce qui tient de l'opacité et de la transparence comme la manière dont les machines permettent d'engager des interactions montrent ainsi que leur action mobilise non seulement l'attention, mais également des représentations du corps. Or cette relation à l'image mimétique, et les processus cognitifs qu'elle implique en matière de perception et de projection, intéresse depuis longtemps l'histoire de l'art.

Il s'agit finalement moins, comme l'a noté Ernst Gombrich, d'un problème relevant du «vrai» ou du «faux» que de la «concordance entre le modèle et l'image reproduite [telle qu'elle] s'obtient peu à peu, au terme d'un long processus dont la durée et les difficultés dépendent du choix d'un schéma initial que l'artiste adapte aux détails caractéristiques de l'image réelle" (Gombrich 2002 : 62). Une image, par nature imprécise, participe ainsi de la définition de ce qu'elle représente. Mais, parallèlement, un spectateur reconnaîtra plus ou moins facilement 


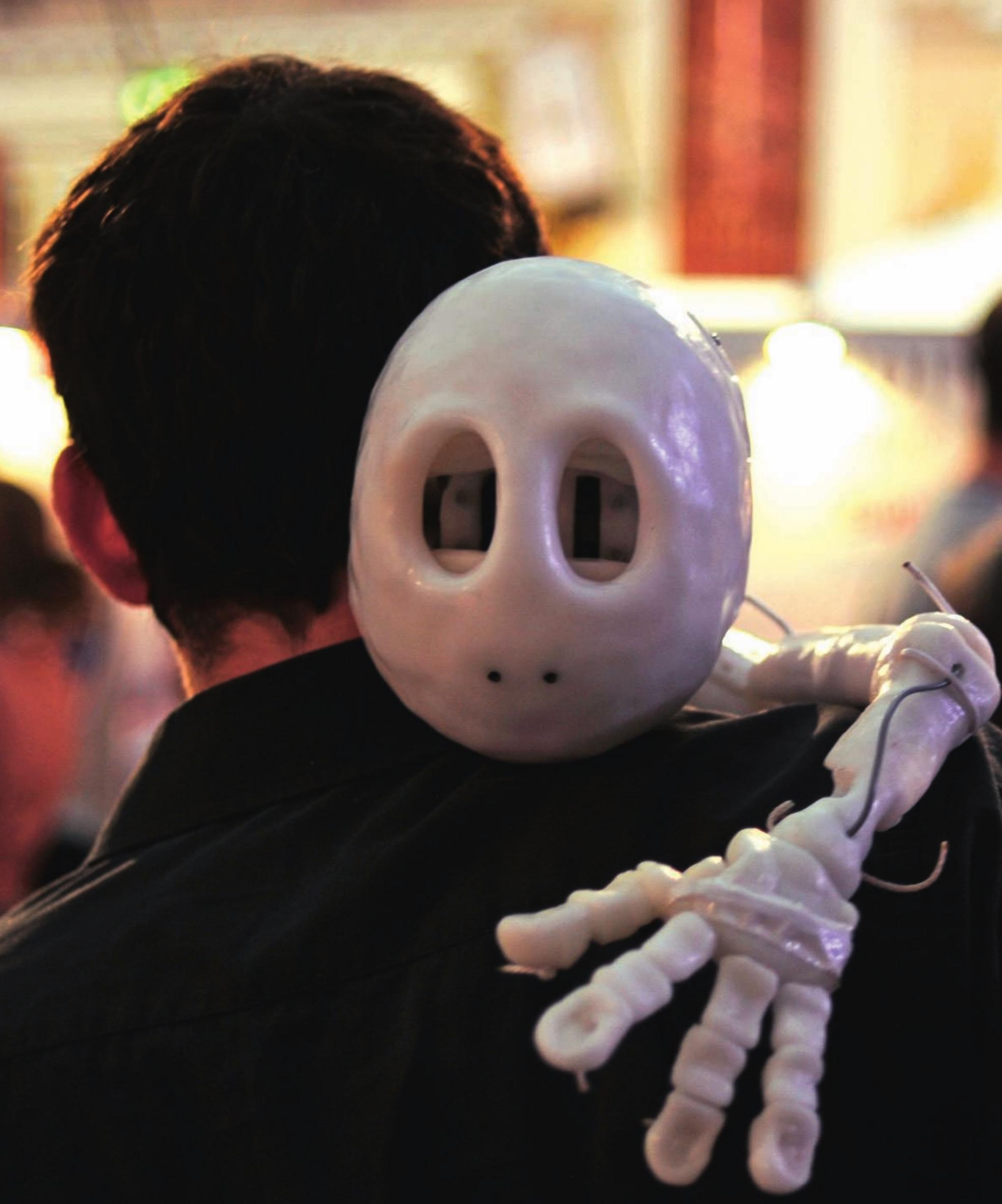




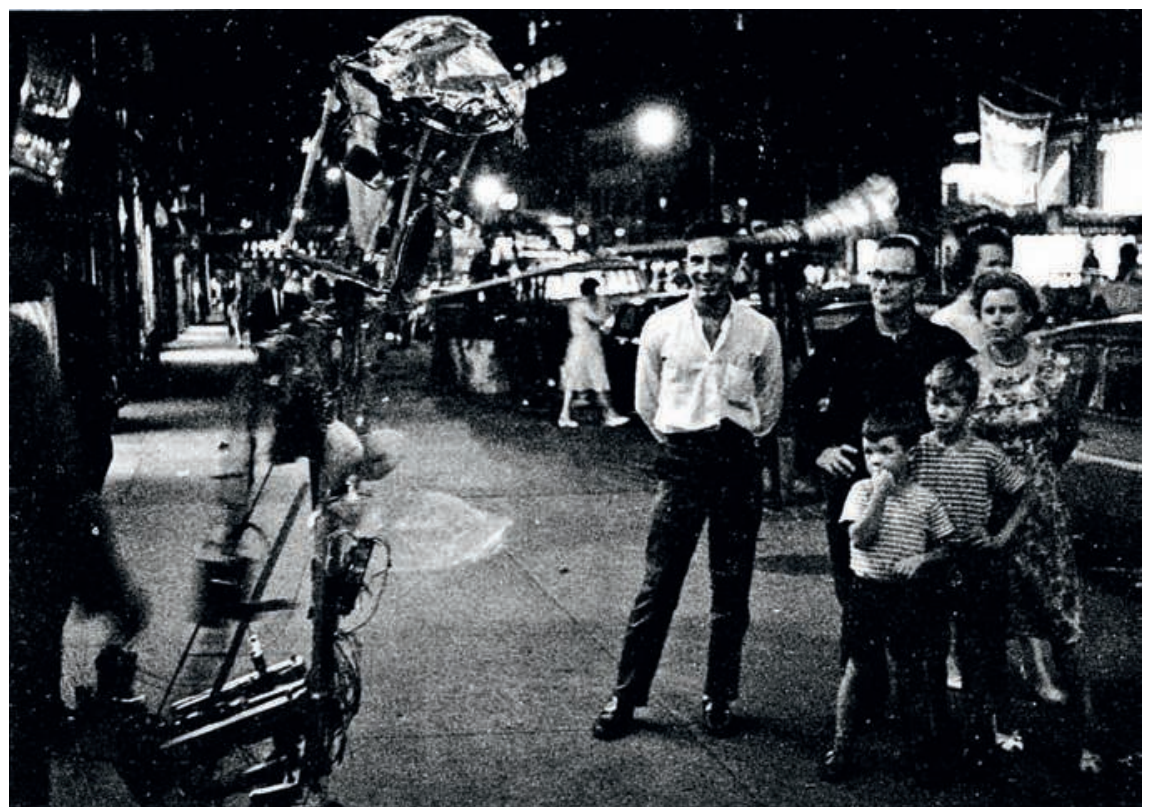

Fig. 10 K-456 au Second Annual New York Avant-Garde Festival, 31 août 1964, DR. Photo @ Estate of Peter Moore/VAGA, NYC.

ce qui est représenté en fonction de la familiarité qu'il entretient avec l'objet en question. Gombrich insiste sur ces deux aspects de l'imitation : notre capacité à rendre compte de la réalité et à projeter sur elle les éléments d'une perception fantôme, prenant part aux processus classificatoire et herméneutique de la mimèsis même. Le spectateur doit d'abord disposer d'une image suffisamment lacunaire, d'un «écran ». Il doit ensuite n'avoir aucun doute sur la manière dont il pourra combler les vides de cette image (ibid. : 174). Si l'on songe aux robots que nous avons présentés, la manière dont quelques éléments suffisent à fonder l'impression (ou le souvenir) d'une présence semble comparable à la façon dont une œuvre d'art, en jouant avec l'ambiguïté formelle, peut orienter l'activité du créateur et celle des spectateurs vers une commune recherche de sens. En imitant ainsi la nature de manière suffisamment vague pour ménager des espaces de projection pour les spectateurs, les robots constitueraient une illustration des formes de socialisation des représentations de la personne. Entre un corps qui trouve dans l'objet à la fois l'ébauche et la confirmation de sa propre mécanicité, et une machine qui trouve dans le corps les principes fondateurs d'une présence qui ne lui est pourtant pas équivalente, cette médiation apparaît bien comme une forme de coopération indirecte, visant à définir la pertinence et les effets de la métaphore mécaniste à travers une mobilisation généralisée des sens ${ }^{17}$.

L'un des principaux enjeux de l'interaction avec les machines anthropomorphes renverrait ainsi à l'évaluation de leur nature même, ou plus précisément à la manière dont celle-ci peut être définie par contraste avec l'expérience ordinaire que l'on fait de notre propre image (Becker 2011). C'est à ce point que la mimèsis scientifique peut retrouver l'imitation de la nature, au travers d'une poétique de la merveille qui, comme déjà par le passé, fait coïncider l'automate merveilleux et l'émerveillement qu'il suscite dans l'œil du spectateur (Brunon 2006). Mais, curieusement, cette action du merveilleux sur l'objet, qu'elle se fonde sur l'ingéniosité du créateur ou sur la réaction du spectateur, n'évacue aucune référence au réel. Au contraire, elle concourt à la vraisemblance, elle 
en renforce l'image; ou, du moins, elle nous engage dans l'image et dans des croyances diverses renvoyant à la manière même dont celle-ci peut être perçue. C'est ce que note Gombrich lorsqu'il souligne qu'en nous imprégnant de la signification de l'élément perçu, la lecture d'une image procède d'une métamorphose; manière, pour reprendre le célèbre exemple, qu'a la figure d'un lapin de se muer, selon la façon dont on l'observe, en celle d'un canard, et réciproquement.

Il semble difficile d'affirmer si un spectateur peut se laisser tromper aussi aisément que durablement par la machine qu'il observe. Les spectateurs passent ainsi un temps très variable à jouer avec les machines d'Ihnatowicz, ils craignent ou espèrent une possible fusion avec l'objet technique sans jamais remettre en cause l'humanité de Stelarc, et considèrent Robot K-456 ou Heart non seulement à travers les indices de leur subjectivité, mais surtout leur étrangeté. Si d'un côté le spectateur semble effectivement disposé à reconnaître et à projeter sur l'action de ces machines des éléments de sa propre humanité, il est en fait difficile d'imaginer que l'image du robot soit dotée d'un pouvoir tel qu'elle fasse passer l'imitation plus ou moins ressemblante du modèle pour le modèle lui-même. C'est, d'une part, toute l'ambiguïté de l'imitation que de produire à la fois un double du modèle et les moyens de dépasser ce dernier; innocente magie, note Louis Marin, dont l'issue est de tromper les yeux de celui qui les pose sur l'objet d'art; image en trompe l'œil d'où résulte l'effet d'une présence stupéfiante et fragile travaillant la représentation par le simulacre ${ }^{18}$ (Marin 1994). C'est, d'autre part, toute l'instabilité de l'organisation formelle des images mimétiques, dont la constitution s'appuie sur sorte de négociation autour des éléments pouvant être extraits de la réalité et, par conséquent, sur un ensemble de projections diverses travaillant la connaissance du corps à la fois par le biais de la technique et celui de l'esthétique.

Ces relations, qui ont la machine pour centre, participent au même titre que l'image d'un événement ontologique (Freedberg 1991: 76-77). Le caractère ${ }^{19}$ dont est doté un robot, malgré l'apparente fragilité des projections qu'il engage, peut d'abord se fonder sur une communauté de formes, comprenant autant son apparence générale que les actes dont il a été rendu capable. Cependant, l'écart persistant entre la machine et l'humain, aisément évalué par le spectateur, constitue un autre aspect de la caractérisation des machines, qui vise alors moins à confirmer la ressemblance au modèle qu'à la mettre à l'épreuve. L'interaction d'un humain et d'un robot n'est en effet pas toujours aussi simple que les roboticiens pourraient le souhaiter. Et elle semble dépendre autant de la manière dont le spectateur peut assimiler les formes et les mouvements à la vie que des interrogations qu'il formule face aux incertitudes devant lesquelles ce genre d'objet le place. Le robot apparait ici comme un voile dressé entre le créateur et le spectateur, sur lequel s'imprime une nature que tous deux partagent sans qu'ils puissent pour autant l'embrasser dans son ensemble. L'isomorphisme limité, lors de l'interaction, aux éléments projetés par le créateur et le spectateur sur l'objet ne semble pas permettre la fusion entre l'image et son prototype. C'est du point de vue adopté que semblent alors dépendre nos relations avec de telles machines, selon que l'on souhaite y voir ici un «canard», là un «lapin ».

EHESS/Laboratoire d'anthropologie sociale joffrey.becker@ehess.fr $\bullet$

18. Le pouvoir véritable du trompel'œil, note Marin, réside dans le retournement de la représentation qu'il est en mesure d'opérer. Capturant ainsi l'attention du spectateur, le trompe-l'œil le transforme en son propre spectacle (Marin 1994 : 311).

19. J'emploie le terme dans un sens proche du terme anglais character.

mots clés / keywords : arts // arts • robotique // robotics • anthropomorphisme // anthropomorphism. 


\section{Bibliographie}

BECKER, Joffrey

2011 «Récursions chimériques. De l'anthropomorphisme des robots autonomes à l'ambiguïté de l'image du corps humain », Gradhiva 13 : 113-129.

BeDAU, Mark A.

1997 «Weak Emergence », Noûs 31, supplément Philosophical Perspectives 11: 375-399.

BoAl, Augusto

1996 Le Théâtre de l'opprimé. Paris,

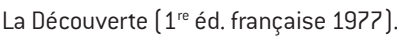

\section{BraCelli, Giovanni Battista}

1624 Bizzarie di varie figure, di Giovanbatista Braccelli, pittore fiorentino, All'Ill.mo S. Don Pietro Medici. Livourne, [s.n].

\section{BROOKs, Rodney}

1990 «Elephants don't play chess », Robotics and autonomous systems 6(1-2) : 3-15.

\section{BRUnon, Hervé}

2006 «Ars naturans, ou l'immanence du principe : l'automate et la poétique de l'illusion au xvI siècle », in Jan Pieper (éd.), Das Château de Maulnes und der Manierismus in Frankreich. Munich-Berlin, Deutscher Kunstverlag : 275-304.

Decker, Edith et Leeber, Irmeline 1993 Nam June Paik. Du cheval à Christo et autres écrits. Bruxelles, Nam June Paik-Éditions Leeber Hossmann.

DeLgado, José M.R.

1969 « Radio stimulation of the brain in primates and man », Anesthesia and analgesia 48[4] : 529-542

Dixon, Steve

2007 Digital Performance. A history of new media in theater, dance, performance art, and installation. Cambridge, MIT Press.

Dutens, Louis

1772 Lettres sur un automate qui joue aux échecs. Paris, [s.n].

FRANKO, Mark

1993 La Danse comme texte. Idéologies du corps baroque. Paris, Kargo/L'Éclat.

\section{FreEDBERG, David}

1991 The Power of images. Study in the history and theory of response. Chicago, University of Chicago Press.

\section{GeLL, Alfred}

1992 « The technology of enchantment and the enchantment of technology », in Jeremy Coote et Anthony Shelton [éd.], Anthropology, art and aesthetics. Oxford, Clarendon Press : 40-63.

1998 Art and agency. An anthropological theory. Oxford, Clarendon Press.

GomBRICH, Ernst H.

2002 L'Art et l'illusion. Psychologie de la représentation picturale. Paris, Phaidon.

Guillot, Agnès et Meyer, Jean-Arcady

2008 La Bionique. Quand la science imite la nature. Paris, Dunod.

HANHARDT, John G.

2006 « Chance in a lifetime: on Nam June Paik », Artforum international $44(8)$; http://artforum.com/inprint/ issue $=200604 \&$ id $=10623$ (consulté le 16 janvier 2012).

\section{Heudin, Jean-Claude}

2008 Les Créatures artificielles. Des automates aux mondes virtuels. Paris, Odile Jacob.

Jones, Amelia et Warr, Tracey

2005 Le Corps de l'artiste. Paris, Phaidon.

KAC, Eduardo

1997 « Origin and development of robotic art 》, Art Journal 56(3) : 60-6?.

\section{KaPlan, Frédéric}

2005 Les Machines apprivoisées. Comprendre les robots de loisir. Paris, Vuibert.

KeEne, Donald

1990 [1965] No and Bunraku. Two forms of japanese theatre. New York, Columbia University Press.

KirBY, Michael

1965 Happenings, an illustrated anthology. New York, E.P. Dutton \& Co. Inc.

Marin, Louis

1994 De la représentation. Paris, GallimardLe Seuil.

\section{Metzner, Paul}

1998 « Robert-Houdin and the vogue of the automaton-builders », Crescendo of the virtuoso. Spectacle, skill, and self-promotion in Paris during the age of Revolution. Berkeley, University of California Press : 160-210.
Monceaux, Jérôme, Becker, Joffrey, Boudier, Céline et Mazel, Alexandre

$2009 \ll$ Demonstration: first steps in emotional expression of the humanoid robot Nao », Proceedings of the 2009 international conférence on multimodal interfaces ICMIMLMI. New York, Association for Computing Machinery : 235-236.

REICHARDT, Jasia 1972 «Art at large », New Scientist 54(794) : 292.

Rigoley de Juvigny, Jean-Antoine 1770 « Lettre de M. Rigoley de Juvigny au sujet de l'automate qui joue aux échecs », Mercure de France : 181-188.

Riskin, Jessica

2003 « The defecating duck, or the ambiguous origins of artificial life », Critical inquiry 29(4) : 599-633.

\section{RoBERT-Houdin, Jean-Eugène} 1859 Confidences d'un prestidigitateur. Une vie d'artiste, t. I. Paris, Librairie nouvelle.

SCHAFFER, Simon

1999 «Enlightened automata », in William Clark et Simon Schaffer (éd.), The Sciences in Enlightened Europe. Chicago, Chicago University Press : 126-165.

SMITH, Marquard

2005 Stelarc. The Monograph. Cambridge, MIT Press.

\section{Suchman, Lucy}

2007 « Demystification and reenchantments of the humanlike machine », in Human-Machine reconfiguration. Plans and situated actions. Cambridge, Cambridge University Press : 241-258.

Suouet, Annie

2006 «Scènes. Le corps dansant : un laboratoire de la perception », in JeanJacques Courtine [éd.], Histoire du corps, vol. III : La Mutation du regard. Paris, Seuil : 393-415.

\section{TuRKLe, Sherry}

2007 Evocative objects. Things we think with . Cambridge, MIT Press.

TURNER, Victor

1987 The Anthroplogy of performance. New York, PAJ Publications. 


\section{VAuCANSON, Jacques de}

1738 « Lettre de M. de Vaucanson à M. l'abbé D. F. », in Le Mécanisme du flûteur automate présenté à messieurs de l'Académie royale des sciences. Paris, Guérin : 19-22.

Windisch, Charles Gotlieb de

1783 Lettres sur le joueur d'échecs de M. de Kempelen. Bâle, [s.n].

\author{
Zivanowic, Aleksandar \\ $2005 \ll$ SAM, the senster and the bandit. \\ Early cybernetic sculptures by Edward \\ Ihnatowicz », Proceedings of the Symposium \\ on robotics, mechatronics and animatronics \\ in the creative and entertainment industries \\ and arts : 1 -?.
}

\section{Résumé / Abstract}

Joffrey Becker, Le corps humain et ses doubles. Sur les usages de la fiction dans les arts et la robotique - Les robots participent sans doute d'une des formes d'anthropomorphisme parmi les plus récentes et les mieux assumées au sein de notre environnement culturel. Ils apparaissent également comme des objets assez peu étudiés par les anthropologues. Ils sont pourtant mobilisés par leurs créateurs au titre d'une réflexion sur notre condition, et ce dans des contextes qui semblent bien distincts: la science et la fiction. Nous proposons ici d'explorer quelques éléments constitutifs des relations entre humains et machines. II s'agit de voir comment le mouvement conjoint d'imitation et de compréhension du corps favorise la création d'un espace relationnel permettant de penser notre humanité et ses transformations. Nous verrons que cette perspective anthropologique traverse la robotique comme les arts.
Joffrey Becker, The human body and its doubles. About the uses of fiction in the arts and robotics - Robots belong to one of the most recent forms of anthropomorphism that is widely accepted in our cultural environment. Yet, anthropologists have tended to understudy them. Robots, however, provide a means for their creators to reflect on our condition in very distinctive contexts: science and fiction. Here, we propose to explore some constitutive aspects of the relationships between humans and machines. The article shows how the combined movement of body imitation and body understanding induces the creation of a relational space in which to think about our humanity and its transformations. This anthropological perspective applies as much to robotics as to the arts. 